10. F. T. Wang, On Riesz summability of Fourier series, Proc. London Math. Soc. (2) vol. 47 (1942) pp. 308-325.

11. - On Riesz summability of Fourier series (III), Proc. London Math. Soc. (2) vol. 51 (1949) pp. 215-231.

National TaIwan University

\title{
ON THE IDENTITY OF FUNCTION SPACES ON CARTESIAN PRODUCT SPACES ${ }^{1}$
}

\author{
JOHN C. HOLLADAY
}

For $i=1, \cdots, n$, let $S_{i}$ be a compact Hausdorff space and $F_{i}$ be a closed linear subspace of the complex Banach space $C\left(S_{i}\right)$, the set of all continuous functions from $S_{i}$ to the complex numbers. Let $S_{1} \times \cdots \times S_{n}$ be the Cartesian Product of $S_{1}, \cdots, S_{n}$.

Define $F_{1} * \cdots * F_{n}$ as $\left\{\phi \mid \phi \in C\left(S_{1} \times \cdots \times S_{n}\right)\right.$; for any $i$ $=1, \cdots, n$ and $\left(s_{1}, \cdots, s_{i-1}, s_{i+1}, \cdots, s_{n}\right), \phi\left(s_{1}, \cdots, s_{i-1}, \cdot\right.$, $\left.\left.s_{i+1}, \cdots, s_{n}\right) \in F_{i}\right\}$. Also, define $F_{1} \otimes \cdots \otimes F_{n}$ as the closure of the space of linear combinations of functions of the form $\phi\left(s_{1}, \cdots, s_{n}\right)$ $=f_{1}\left(s_{1}\right) \times \cdots \times f_{n}\left(s_{n}\right)$ where each $f_{i} \in F_{i}$, where we base the topology on the norm, $\|\phi\|=\max _{s_{1}}, \ldots, s_{n}\left|\phi\left(s_{1}, \cdots, s_{n}\right)\right|$.

It is easily shown that $F_{1} \otimes \cdots \otimes F_{n}$ is a subspace of $F_{1} * \cdots * F_{n}$ and that if $F_{2}$ is one-dimensional, then $F_{1} \otimes F_{2}=F_{1} * F_{2}$. Furthermore, by using continuous partitions of unity, one may show that $F_{1} \otimes C\left(S_{2}\right)$ $=F_{1} * C\left(S_{2}\right)$. Therefore, if all but at most one of the $F_{i}$ are either onedimensional or $C\left(S_{i}\right)$, then $F_{1} \otimes \cdots \otimes F_{n}=F_{1} * \cdots * F_{n}$. However, it is not known whether for all cases $F_{1} \otimes \cdots \otimes F_{n}$ will equal $F_{1} * \cdots * F_{n}$ or not. ${ }^{2}$ Although this question is not fully answered here, the purpose of this paper is to give a partial answer to this question. The results and arguments of this paper also apply to realvalued function spaces.

1. Lemma. Let $F$ be a closed linear subspace of $C\left(S_{1}\right)$ and $G$ a closed linear subspace of $C\left(S_{2}\right)$. Let $H$ be a closed subspace of $G$ differing from $G$ by only one dimension. Then $F \otimes G=F * G$ implies that $F \otimes H=F * H$.

Received by the editors October 22, 1956 and, in revised form, April 16, 1957.

1 A small part of the work done under an AEC Predoctoral Fellowship at Yale University, year 1952-1953, under the kind and patient guidance of Dr. Charles E. Rickart.

2 Under Proposition 37 of his first paper of the Amer. Math. Soc. Memoirs, no. 16, Alexandre Grothendieck discusses a number of conjectures which are equivalent to this one. 
Proof. Choose a continuous linear functional $x^{*}$ on $G$ such that $\left\|x^{*}\right\|=1$ and $x^{*}(h)=0$ for $h \in H$. Choose $g_{0} \in G$ such that $x^{*}\left(g_{0}\right)=1$. Then every element of $G$ will be of the form $h+\lambda g_{0}$ where $h \in H$ and $\lambda$ is a complex number.

Choose $\phi \in F * H$ and $\epsilon>0$. Since $\phi \in F * G=F \otimes G$, choose $f_{i} \in F$, $g_{i} \in G, i=1, \cdots, k$ such that

$$
\left\|\phi-\sum_{i=1}^{k} f_{i} \times g_{i}\right\|<\epsilon\left(1+\left\|g_{0}\right\|\right)^{-1} .
$$

However, each $g_{i}$ may be expressed as $h_{i}+\lambda_{i} g_{0}$ where $h_{i} \in H$. There fore,

$$
\sum_{i=1}^{k} f_{i} \times g_{i}=\sum_{i=1}^{k} f_{i} \times\left(h_{i}+\lambda_{i} g_{0}\right)=y+z \times g_{0}
$$

where $y \in F \otimes H$ and $z \in F$.

For each $s \in S_{1}$,

$$
\begin{aligned}
|z(s)| & =\left|x^{*}\left[z(s) g_{0}\right]\right|=\mid x^{*}\left[y(s, \cdot)+z(s) g_{0}-\phi(s, \cdot)^{l}\right. \\
& \leqq\left\|y+z \times g_{0}-\phi\right\|\left\|x^{*}\right\|<\epsilon\left(1+\left\|g_{0}\right\|\right)^{-1} .
\end{aligned}
$$

Therefore,

$$
\begin{aligned}
& \left\|\sum_{i=1}^{k} f_{i} \times h_{i}-\phi\right\|=\|y-\phi\| \\
& \quad \leqq\left\|y+z \times g_{0}-\phi\right\|+\left\|z \times g_{0}\right\|=\left\|y+z \times g_{0}-\phi\right\|+\|z\|\left\|g_{0}\right\| \\
& \quad<\epsilon\left(1+\left\|g_{0}\right\|\right)^{-1}+\epsilon\left\|g_{0}\right\|\left(1+\left\|g_{0}\right\|\right)^{-1}=\epsilon .
\end{aligned}
$$

Since $f_{i} \in F_{i}$ and $h_{i} \in H$, it follows that $\phi \in F \otimes H$. Therefore, $F \otimes H$ $=F * H$.

2. Lemma. Let $S_{1}, S_{2}, F, G$ and $H$ be as in Lemma 1. Then $F \otimes H$ $=F * H$ implies that $F \otimes G=F * G$.

Proof. Choose $g_{0} \in G-H$ and $x^{*}$ as in the proof of Lemma 1 . Since the set of continuous linear functionals on $C\left(S_{2}\right)$ is the set of regular measures on $S_{2}$, we may choose a measure $\mu$ such that $\int_{S_{2}} g\left(s_{2}\right) \mu\left(d s_{2}\right)=x^{*}(g)$ for each $g \in G$.

Consider an arbitrary element $\phi \in F * G$. For $s_{1} \in S_{1}$, define $\psi\left(s_{1}\right)$ as

$$
\int_{S_{2}} \phi\left(s_{1}, s_{2}\right) \mu\left(d s_{2}\right)
$$


Let $\mu^{\prime}$ be any regular measure on $S_{1}$ such that for $f \in F, \int_{S_{1}} f\left(s_{1}\right) \mu^{\prime}\left(d s_{1}\right)$ $=0$. Then using Fubini's Theorem, we get that

$$
\begin{aligned}
\int_{S_{1}} \psi\left(s_{1}\right) \mu^{\prime}\left(d s_{1}\right) & =\int_{S_{1}} \int_{S_{2}} \phi\left(s_{1}, s_{2}\right) \mu\left(d s_{2}\right) \mu^{\prime}\left(d s_{1}\right) \\
& =\int_{S_{2}} \int_{S_{1}} \phi\left(s_{1}, s_{2}\right) \mu^{\prime}\left(d s_{1}\right) \mu\left(d s_{2}\right)=\int_{S_{2}} 0 \mu\left(d s_{2}\right)=0 .
\end{aligned}
$$

Therefore, $\psi \in F$.

For any $s_{1} \in S_{1}$, it follows that

$$
\int_{S_{2}}\left[\phi\left(s_{1}, s_{2}\right)-\psi\left(s_{1}\right) g_{0}\left(s_{2}\right)\right] \mu\left(d s_{2}\right)=\psi\left(s_{1}\right)-\psi\left(s_{1}\right)=0
$$

and so $\phi\left(s_{1}, \cdot\right)-\psi\left(s_{1}\right) g_{0}(\cdot)$ is an element of $H$. Therefore $\phi-\psi \times g_{0}$ $\in F * H=F \otimes H C F \otimes G$. Consequently, $\phi=\left[\phi-\psi \times g_{0}\right]+\psi \times g_{0}$ is an element of $F \otimes G$.

3. Theorem. For each $i=1, \cdots, n$, let $G_{i}$ and $F_{i}$ be closed linear subspaces of $C\left(S_{i}\right)$ which differ from each other by at most a finite number of dimensions. Let no $F_{i}$ or $G_{i}$ be zero-dimensional.

Then $F_{1} \otimes \cdots \otimes F_{n}=F_{1} * \cdots * F_{n}$ if and only if $G_{1} \otimes \cdots \otimes G_{n}$ $=G_{1} * \cdots * G_{n}$.

Proof. Let $H_{1}, \cdots, H_{k}$ be closed linear subspaces of $C\left(S_{1}\right)$ such that $H_{1}=F_{1}, H_{k}=G_{1}$ and for $i=1, \cdots, k-1, H_{i}$ and $H_{i+1}$ differ from each other by only one dimension. If $F_{1} \otimes \cdots \otimes F_{n}$ $=F_{1} * \cdots * F_{n}$, then $F_{2} \otimes \cdots \otimes F_{n}=F_{2} * \cdots * F_{n}$. Using Lemma 1 or 2 , we see that $H_{2} \otimes F_{2} \otimes \cdots \otimes F_{n}=H_{2} * F_{2} * \cdots * F_{n}$. Applying the lemmas in this way, a total of $k-1$ times, we see that $G_{1} \otimes F_{2} \otimes \cdots \otimes F_{n}=G_{1} * F_{2} * \cdots * F_{n}$. Now repeating this procedure $n-1$ more times, we obtain that $G_{1} \otimes \cdots \otimes G_{n}=G_{1} * \cdots * G_{n}$.

By repeating the above arguments, we see that $G_{1} \otimes \cdots \otimes G_{n}$ $=G_{1} * \cdots * G_{n}$ implies $F_{1} \otimes \cdots \otimes F_{n}=F_{1} * \cdots * F_{n}$ and hence the theorem is proved.

Los Alamos Scientific Laboratory 